\title{
Multidetector Computed Tomography Evaluation of Omental Infarct
}

\author{
Merin Babu ${ }^{1}$ Rohini Avantsa ${ }^{1}$
}

Address for correspondence Merin Babu, MBBS, Department of Radiodiagnosis, KS Hegde Medical Academy, Nitte deemed to be University, Mangalore-575018, India (e-mail: merinbabu09@gmail.com).

\begin{abstract}
Background Omental infarction is a rare cause of acute pain abdomen and is difficult to diagnose clinically due to nonspecific clinical features. Multidetector computed tomography (MDCT) imaging of the abdomen is essential for the diagnosis of omental infarction and is also crucial to rule out appendicitis and other causes of acute pain abdomen.

Objective The main purpose of this article is to describe the MDCT features of omental infarction in clinching the diagnosis and differentiating it from its clinical mimics.

Methods CT findings of five patients with diagnosed omental infarction were retrospectively reviewed from 2014 to 2019. All patients presented with pain abdomen on the right side of the abdomen with various clinical diagnoses. MDCT was done for diagnosis and features evaluated include size of the lesion, location, relation to colon, and findings that were correlated to clinical presentation and etiology.

Results Age distribution ranged between 36 and 55 years with female predominance. Out of five cases, one was secondary omental infarction due to inguinal hernia causing omental torsion and the other four cases were of unknown etiology. Three cases were managed conservatively and the other two cases were surgically managed. Three lesions

Keywords

- omental infarction

- acute appendicitis

- computed tomography

- whirl sign were in right iliac fossa and two lesions were in the supraumbilical region on the right side. Conclusion Omental infarction should be considered important in the differential diagnosis of acute pain abdomen mimicking acute appendicitis and requires $\mathrm{CT}$ abdomen to establish diagnosis. Knowledge of typical imaging findings and application for diagnosis are important, as it is a self-limiting disease and can avoid unnecessary surgical interventions.
\end{abstract}

\section{Introduction}

Omental infarction is a rare cause of acute abdominal pain and clinical diagnosis is challenging due to its relatively low incidence and nonspecific presentation. Omental infarct typically presents with right lower abdominal pain, vomiting, low-grade fever, and occasionally with a palpable abdominal mass. Although clinically it is indistinguishable from acute appendicitis and cholecystitis, ${ }^{1}$ imaging findings and radiological diagnosis are more promising due to advancements in technology in the recent past. Omental infarct occurs due to vascular compromise of the greater omentum and

Dol https://doi.org/

10.1055/s-0039-3402631

ISSN 2581-9933. is classified as primary or secondary. Secondary omental infarction is caused due to adhesion between the omentum and pathological foci (hernia, surgical scar, and tumors), hypercoagulable state, and mesenteric vein thrombosis due to right heart failure. ${ }^{2}$ If no cause is identified, then it is called idiopathic or primary omental infarction that is likely due to anatomical variations like bifid, bulky or redundant omentum. The diagnosis of omental infarction is primarily based on computed tomography (CT) that allows conservative management. About 400 cases of omental infarction were reported in literature; however, there is a paucity of larger case series describing the imaging appearance. ${ }^{3}$ 


\section{Aims and Objectives}

The main purpose of this article is to describe the multidetector computed tomography (MDCT) features of omental infarction in clinching the diagnosis and differentiating from other causes of acute pain abdomen.

\section{Methods}

This is a retrospective review conducted from 2014 to 2019, of five patients with MDCT diagnosis of omental infarction who had been referred to the department of radiology with right lower and/or upper quadrant abdominal pain and with various clinical diagnoses. Basic demographic data of the patients and clinical diagnoses were noted. Ultrasound scans were unremarkable.

\section{MDCT Technique}

All patients underwent CT evaluation on a 16 slice MDCT scanner (General Electric Medical Systems, Milwaukee, Wisconsin, United States) utilizing our contrast-enhanced routine abdomen and pelvis protocol. Axial $5 \mathrm{~mm}$ thick sections were taken from the lung bases to the pubic symphysis after injecting intravenously with 80 to $100 \mathrm{~mL}$ of nonionic iodinated contrast (Omnipaque-300 $\mathrm{mg} / \mathrm{mL}$ ) at a rate of 2 to $3 \mathrm{~mL} / \mathrm{s}$. Oral or rectal contrast was not administered in all five cases as CT scan was done on an emergency basis.

All five patients had noncontrast $\mathrm{CT}$ and at least one contrast-enhanced CT in the portal venous phase (60s). The images were reconstructed utilizing multiplanar reformatted images in sagittal and coronal planes using $1.5 \mathrm{~mm}$ section thickness and viewed in multiple planes.

\section{CT Findings}

Imaging features such as size of the lesion, location, absence of rim enhancement, relation to colon, and bowel wall thickening were included. CT findings were later correlated with postoperative findings in two cases.

\section{Results}

\section{Demographic Data with Clinical Presentation}

Among the five patients, three were females and two were males with an age range of 36 to 55 years (-Table $\mathbf{1}$ ). Pain was unrelated to meal intake. On physical examination, there was tenderness on light palpation localized to right upper/lower abdominal quadrant with guarding. No rebound tenderness was found and Murphy sign was negative. Other systemic examination was unremarkable. Complete blood count, hepatic function tests, lipase, urinalysis, and lactate levels were within normal limits. The electrocardiogram showed normal sinus rhythm. Three cases were clinically diagnosed as acute appendicitis and two cases were diagnosed as acute cholecystitis ( - Table 2 ).

\section{MDCT Features}

MDCT findings consisted of an ill-defined round-oval, heterogeneous fat-density lesion with no definable continuous peripheral rim within the greater omentum in all cases (-Figs. 1 and 2 ). Three cases were in the right iliac fossa and

Table 1 Demographic data of patients

\begin{tabular}{|l|l|}
\hline Gender presentation & 2 \\
\hline Males & 3 \\
\hline Females
\end{tabular}

Table 2 Clinical presentation with clinical diagnosis

\begin{tabular}{|l|l|l|}
\hline Clinical presentation & Clinical diagnosis & Cases \\
\hline Right lower abdominal pain & Acute appendicitis & 3 \\
\hline Right upper abdominal pain & Acute cholecystitis & 2 \\
\hline
\end{tabular}

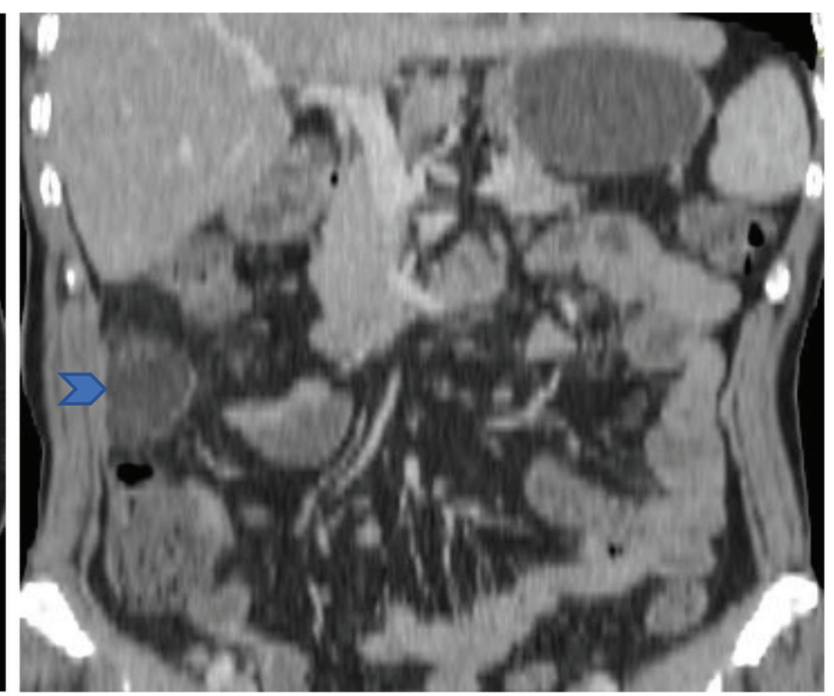

Fig. 1 A 46-year-old man with acute right iliac fossa (RIF) pain. Axial and coronal contrast-enhanced computed tomography images of the abdomen show an oval fat density lesion (arrowhead) in the RIF with a broad base toward parietal peritoneum. The lesion is noted between the ascending colon and the anterior abdominal wall. 
two cases were in the supraumbilical region on the right side ( $\mathbf{- F i g s . ~} 2$ and $\mathbf{3}$ ). The lesions were related to cecum, ascending or transverse colon with no obvious bowel wall thickening in all five patients (-Table 3). Out of five cases, four cases were of primary infarct and one case was secondary infarct ( - Table 4 ) that presented as right inguinal hernia with omental torsion ( - Fig. 4). Omental torsion on MDCT showed fat density lesion with whirling pattern of omental vessels called the whirl sign ( - Fig. 5).

\section{Management}

Three cases were managed conservatively and the other two cases were surgically managed with histopathological diagnosis proven to be omental infarction in operated cases.

\section{Discussion}

Omental infarction was first described by Eithel in the year 1899. ${ }^{4}$ The greater omentum is a large peritoneal fold and is continuous with visceral peritoneum of stomach and transverse colon. It contains fat and blood vessels ( - Fig. $\mathbf{6}$ ). Omental infarct is rare due to extensive vascular collaterals to greater omentum by epiploic arteries. However, as reported in the literature by Park et al, $88 \%$ of infarction commonly occurs on the right side, likely due to the possibility of long and mobile omentum. ${ }^{3}$ Rarely the omental infarction can present on the left side or in the epigastric region..$^{5}$ In our study, three cases presented in the right iliac fossa and the other two cases in the supraumbilical region.
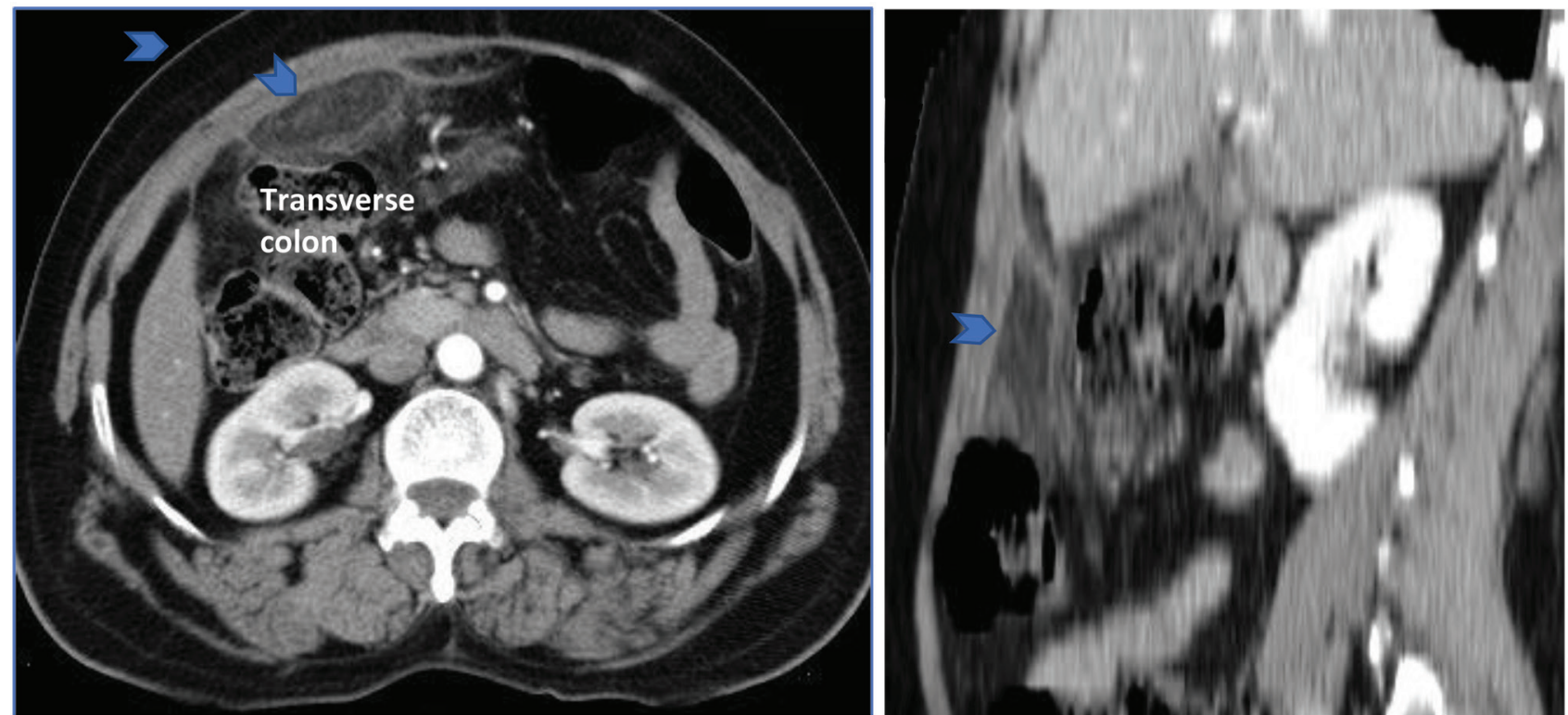

Fig. 2 Axial and sagittal contrast-enhanced computed tomography images of the abdomen in the subhepatic region show a fat density lesion (arrowhead) with hyperattenuating streaky infiltration between the abdominal wall and transverse colon.
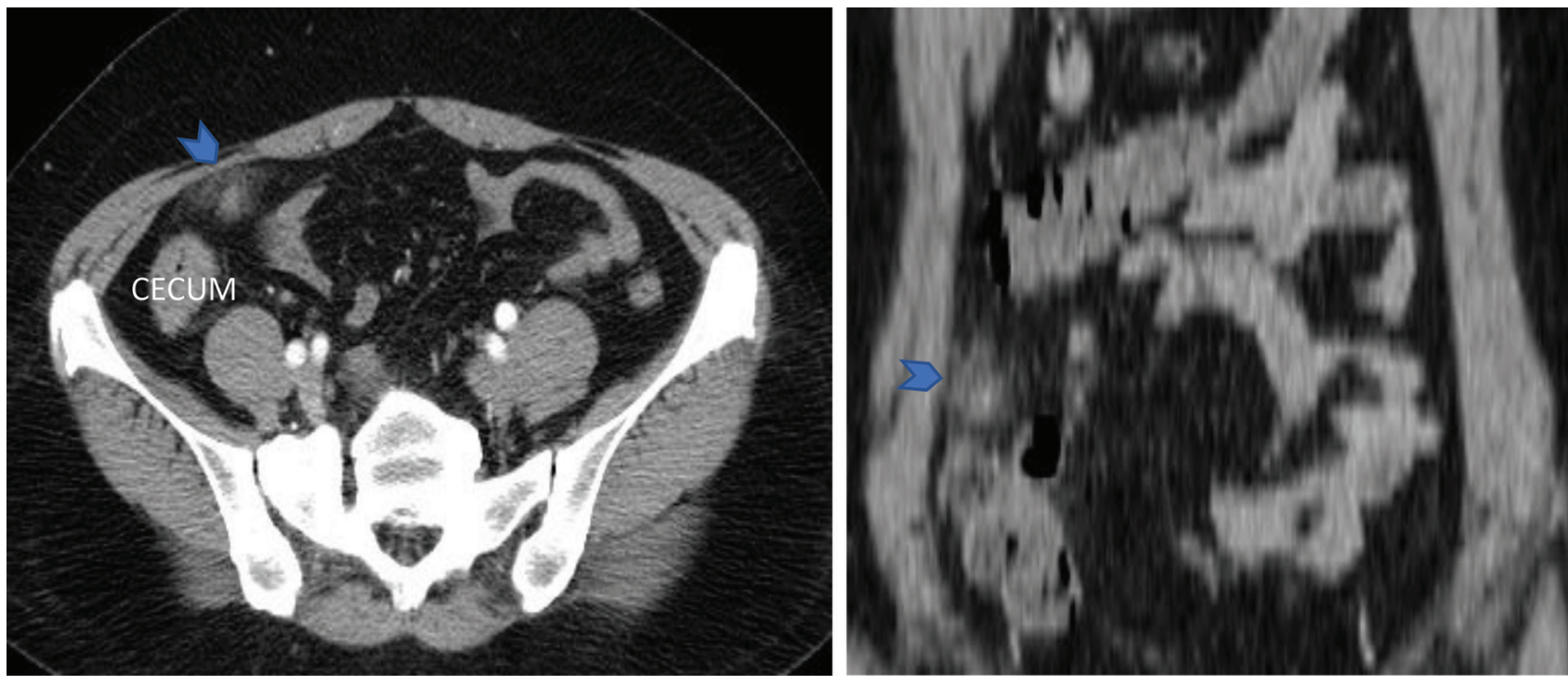

Fig. 3 Axial contrast-enhanced computed tomography abdomen of a 49-year-old female presenting with acute right iliac fossa (RIF) pain shows an ill-defined heterogeneously enhancing small fat density mass in RIF (arrowhead) abutting the parietal peritoneum. 
In $85 \%$ of all cases of omental infarction reported in literature, it was found to occur commonly in adults (40-50 years) presenting as acute or subacute right upper or lower quadrant pain. ${ }^{3}$ In our study, all cases presented with acute pain abdomen (1 day) on the right side. Based on the clinical presentation, most of the cases were diagnosed as acute appendicitis or acute cholecystitis ${ }^{6}$ similar to our study.

Table 3 MDCT features of omental infarct

\begin{tabular}{|c|c|}
\hline \multicolumn{2}{|l|}{ Location of the omental infarct } \\
\hline RIF & 3 \\
\hline Supraumbilical region & 2 \\
\hline \multicolumn{2}{|c|}{ Location of omental infarct in relation to colon/bowel } \\
\hline Ascending colon & 1 \\
\hline Transverse colon & 2 \\
\hline Cecum & 2 \\
\hline \multicolumn{2}{|l|}{ Size of lesion } \\
\hline$>5 \mathrm{~cm}$ & 4 \\
\hline$<2 \mathrm{~cm}$ & 1 \\
\hline \multicolumn{2}{|l|}{ Morphology of omental infarct on CT } \\
\hline In greater omentum & \multirow[t]{4}{*}{ In all cases } \\
\hline Round to oval & \\
\hline Fat density lesion & \\
\hline No definable continuous peripheral rim & \\
\hline
\end{tabular}

Abbreviations: MDCT, multidetector computed tomography; RIF, right iliac fossa

Table 4 Etiology for omental infarct

\begin{tabular}{|l|l|}
\hline Secondary infarct (right inguinal hernia) & $\mathbf{1}$ \\
\hline Primary omental infarct & 4 \\
\hline
\end{tabular}

Omental infarction was classified into two types as primary and secondary infarction ( - Table 5 ) by Leitener et al in 1952 and has been cited in many studies. ${ }^{7}$ Secondary omental infarction is caused due to torsion of omental vessels caused by adhesion between the omentum and pathological foci (hernia, surgical scar, and tumors), hypercoagulable state and mesenteric vein thrombosis due to right heart failure. ${ }^{2}$ If no cause was identified, then it is called idiopathic or primary omental infarction that is likely due to anatomical variations such as bifid, bulky, or redundant omentum. None of the patients in our study had any evidence of hypercoagulable state or previous surgery. One of the patients in our study was diagnosed with omental torsion that was secondary to right inguinal hernia. The rest of the patients (80\%) had no identifiable cause and were diagnosed as primary omental infarction.

The primary modality for the diagnosis of omental infarction is CT that shows a large $(>5 \mathrm{~cm})$ triangular or oval heterogeneous fat density lesion with no enhancing rim or central hyperattenuating foci, which is centered in the greater omentum located between anterior abdominal wall and colon. ${ }^{5,8,9}$ In our study, the fatty lesion was $>5 \mathrm{~cm}$ in most of the cases (80\%) and no evidence of bowel wall thickening was found in all cases. However, rarely there may be bowel wall thickening adjacent to an area of omental infarction due to reactive bowel wall changes. ${ }^{10}$

Omental torsion in CT shows "whirl sign" that appears as streaks due to whirling of omental vessels in concentric pattern. ${ }^{11}$ The whirl sign is a sensitive indication of rotation of the mesentery and vessels but is not specific to omental torsion alone and can be seen in small bowel obstruction, intestinal malrotation, or volvulus. ${ }^{12}$ In our study, whirl sign was seen in one case due to omental torsion secondary to inguinal hernia (-Fig. 5).

Imaging differential diagnosis on $\mathrm{CT}$ for omental fat stranding with acute pain abdomen includes epiploic appendagitis. Epiploic appendagitis, presents as a fatty paracolonic
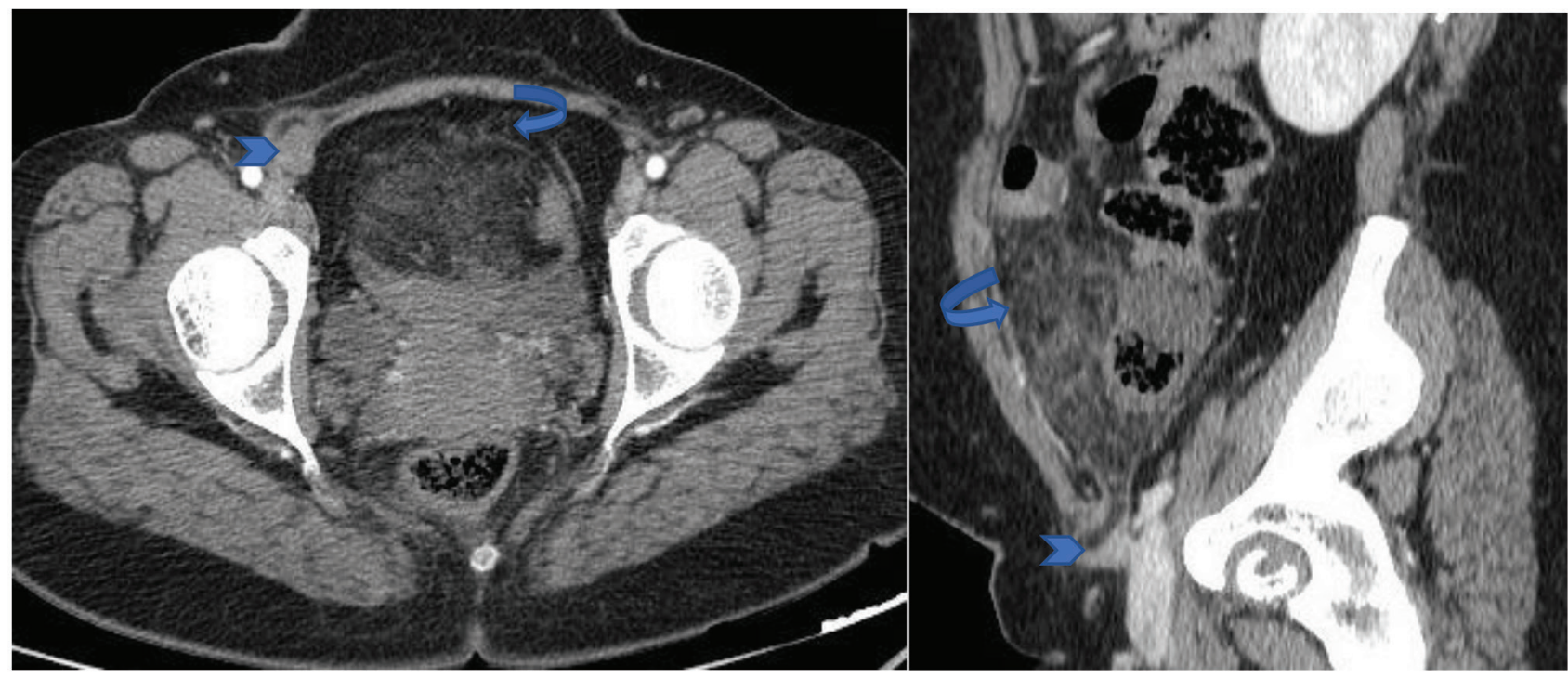

Fig. 4 Secondary omental infarction: A 36-year-old lady presenting with acute right iliac fossa (RIF) pain. Axial and sagittal contrast-enhanced computed tomography abdomen images: Areas of fat density (curved arrow) seen in the RIF herniating into right inguinal region with round ligament (arrowhead) and infarcted omentum as content. Omental infarction was caused secondary to an inguinal hernia. 


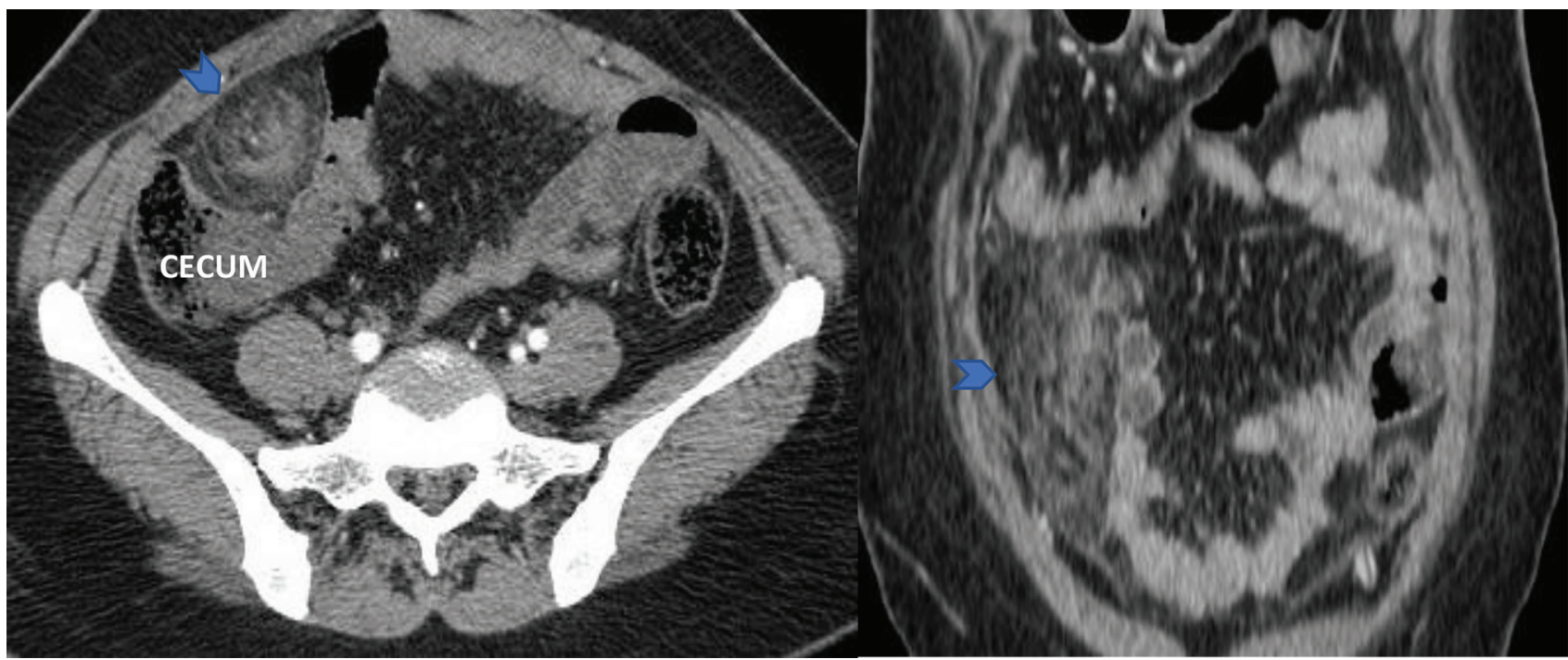

Fig. 5 Axial and coronal contrast-enhanced computed tomography abdomen images of Omental torsion: Hazy omentum with concentric hyperdensity and whirling pattern in right iliac fossa (arrowhead) and abutting the cecum posteriorly. It is called the "Whirl sign."

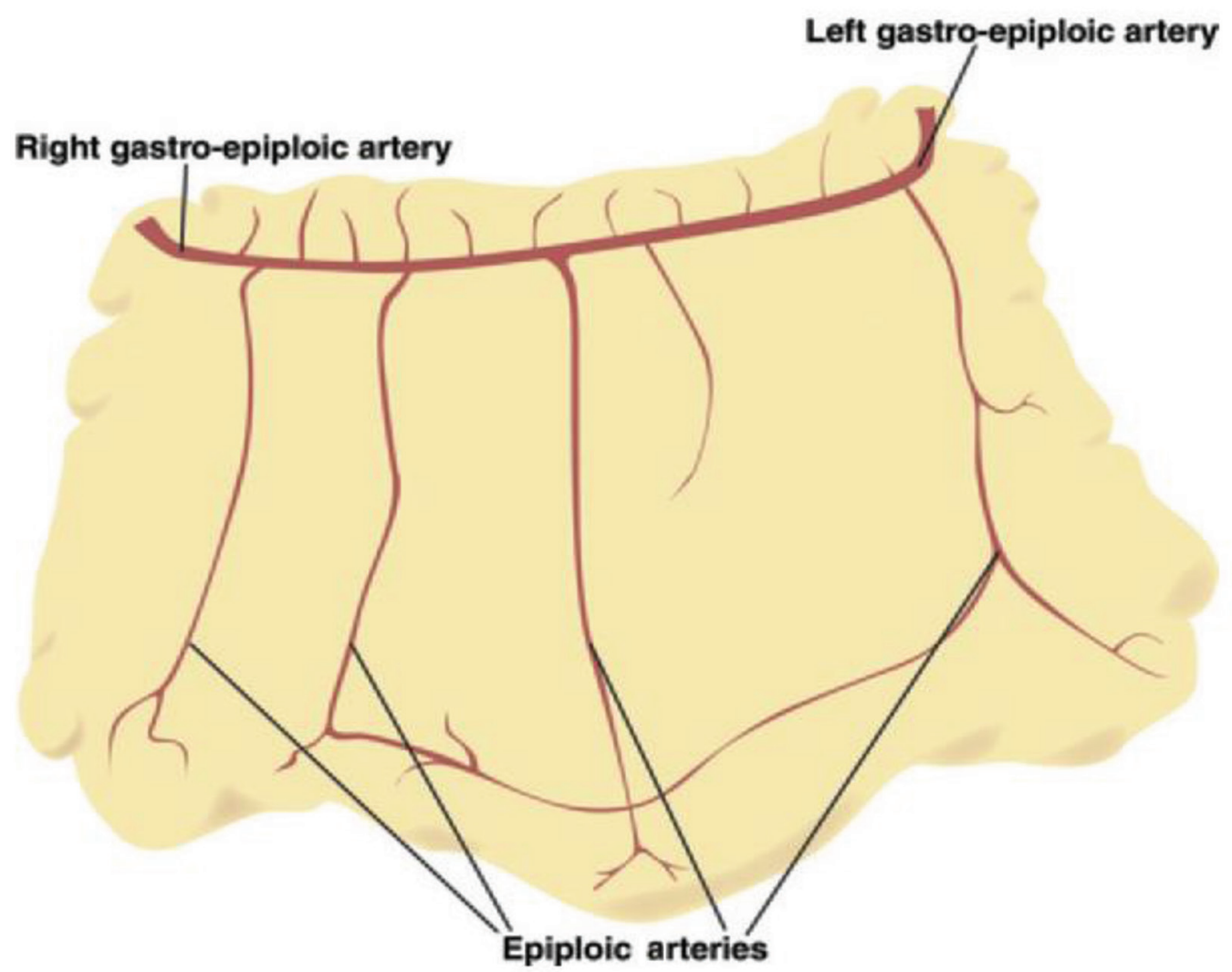

Fig. 6 The right and left gastroepiploic arteries provide blood supply to the greater omentum. The right and left gastroepiploic arteries anastomose within the two layers of the anterior greater omentum along the greater curvature of the stomach. Image courtesy: ESGAR $2019 .{ }^{13}$

oval mass on the left side, is usually $<2 \mathrm{~cm}$ in size with central high attenuation focus representing thrombosed vein and surrounded by hyperdense rim. ${ }^{13}$ There was no evidence of high attenuation peripheral or central foci in any of our five cases.

Usually, conservative treatment is recommended for omental infarction with analgesics and anti-inflammatory drugs, as it is a self-limited disease. However, clinicians should be alert for rare complications such as intestinal obstruction, adhesion or abscess formation, which may need surgical exploration. ${ }^{5}$ Two cases were surgically managed in our study, namely the one case with omental torsion secondary to inguinal hernia and the other case with right iliac fossa fat density lesion with severe intolerable abdominal pain. 
Table 5 Causes of omental infarction

\begin{tabular}{|l|l|}
\hline Primary omental infarction & $\begin{array}{l}\text { Secondary omental } \\
\text { infarction }\end{array}$ \\
\hline $\begin{array}{l}\text { Anatomical variants-bifid } \\
\text { omentum }\end{array}$ & Adhesions (hernia, surgery) \\
\hline Bulky or redundant omentum & $\begin{array}{l}\text { Vasculitis and hypercoagulable } \\
\text { state }\end{array}$ \\
\hline Obesity & Omental cyst/tumor \\
\hline Overeating & Internal or external hernia \\
\hline Local trauma & Diverticulitis \\
\hline Excessive exercise, cough & Congestive heart failure \\
\hline Use of laxatives & \\
\hline
\end{tabular}

\section{Conclusion}

Omental infarction should be considered in the differential diagnosis of acute pain abdomen mimicking acute appendicitis, acute cholecystitis, diverticulitis, etc. MDCT features such as oval heterogeneous fat density lesion in the greater omentum with no high attenuation peripheral or central foci clinch the diagnosis of omental infarction radiologically and avoid unnecessary surgery. Severe pain in the abdomen can be distressing and typical imaging features of omental infarction allay anxiety and allow to treat conservatively as it is a self-limiting disease. Hence, awareness of the imaging features of acute omental infarction is important.

\section{Conflict of Interest}

None declared.

\section{References}

1 Yoo E, Kim JH, Kim MJ, et al. Greater and lesser omenta: normal anatomy and pathologic processes. Radiographics 2007;27(3):707-720

2 Houben $\mathrm{CH}$, Powis M, Wright VM. Segmental infarction of the omentum: a difficult diagnosis. Eur J Pediatr Surg 2003;13(1):57-59
3 Park TU, Oh JH, Chang IT, et al. Omental infarction: case series and review of the literature. J Emerg Med 2012;42(2):149-154

4 EithelG. Rare omental infarction. NYMed Rec 1899;55:715-716

5 Singh AK, Gervais DA, Lee P, et al. Omental infarct: CT imaging features. Abdom Imaging 2006;31(5):549-554

6 Miguel Perelló J, Aguayo Albasini JL, Soria Aledo V, et al. [Omental torsion: imaging techniques can prevent unnecessary surgical interventions]. Gastroenterol Hepatol 2002;25(8):493-496

7 Leitner MJ, Jordan CG, Spinner MH, Reese EC. Torsion, infarction and hemorrhage of the omentum as a cause of acute abdominal distress. Ann Surg 1952;135(1):103-110

8 Goti F, Hollmann R, Stieger R, Lange J. Idiopathic segmental infarction of the greater omentum successfully treated by laparoscopy: report of case. Surg Today 2000;30(5):451-453

9 Pereira JM, Sirlin CB, Pinto PS, Jeffrey RB, Stella DL, Casola G. Disproportionate fat stranding: a helpful CT sign in patients with acute abdominal pain. Radiographics 2004;24(3):703-715

10 Garant M, Taourel P, Fried GM, Bret PM. Thickening of the transverse colon associated with torsion of the greater omentum. AJR Am J Roentgenol 1995;165(5):1309

11 Tandon AA, Lim KS. Torsion of the greater omentum: a rare preoperative diagnosis. Indian $\mathrm{J}$ Radiol Imaging 2010;20(4):294-296

12 Jain P, Chhabra S, Parikh K, Vaidya A. Omental torsion. J Indian Assoc Pediatr Surg 2008;13(4):151-152

13 Leidersnaider C, Perotta R, Araujo S, et al. Do not underestimate the omentum! An illustrated guide to omental inforcts. Poster presented at European Society of Gastrointestinal and Abdominal Radiology, 30th Annual Meeting; 2019

14 Singh AK, Gervais DA, Hahn PF, Sagar P, Mueller PR, Novelline RA. Acute epiploic appendagitis and its mimics. Radiographics 2005;25:1521-1534 